\title{
A Model of Extended, Semisystematic Visual Search
}

\author{
Brian J. Melloy, Sourav Das, Anand K. Gramopadhye, and Andrew T. Duchowski, \\ Clemson University, Clemson, South Carolina
}

\begin{abstract}
Objective: A model of semisystematic search was sought that could account for both memory retrieval and other performance-shaping factors. Background: Visual search is an important aspect of many examination and monitoring tasks. As a result, visual search performance has been the topic of many empirical investigations. These investigations have reported that individual search performance depends on participant factors such as search behavior, which has motivated the development of models of visual search that incorporate this behavior. Search behavior ranges from random to strictly systematic; variation in behavior is commonly assumed to be caused by differences in memory retrieval and search strategy. Methods: This model ultimately took the form of a discrete-time nonstationary Markov process. Results: It yields both performance and process measures that include accuracy, time to perception, task time, and coverage while avoiding the statistical difficulties inherent to simulations. In particular, it was seen that as the search behavior becomes more systematic, expected coverage and accuracy increase while expected task time decreases. Conclusion: In addition to explaining these outcomes and their interrelationships from a theoretical standpoint, the model can predict these outcomes in practice to a certain extent as it can create an envelope defined by best- and worst-case search performance. Application: The model also has the capability of supporting assessment. That is, it can be used to assess the effectiveness of an individual's search performance, and to provide possible explanations for this performance, through the use of one or more of the output measures.
\end{abstract}

\section{INTRODUCTION}

Human visual search is an important aspect of many civilian and military applications such as reconnaissance, tracking, information retrieval, aircraft inspection, medical image screening, industrial inspection, and the monitoring of sonar, radar, and other displays. Even in instances where automation has replaced the human eye as the primary search instrument, the information is frequently still transferred to a human thorough a visual link. Thus, interest in the performance of humans in visual search tasks persists.

In the context of this research, visual search is considered to be an extended examination of a field with many elements (as opposed to those with a small number of visual elements requiring few if any eye movements; e.g., Eriksen, 1990).
An extended search proceeds as a succession of focused gazes or fixations in the person's effort to perceive a target. The performance of such a search is measured by the accuracy achieved - that is, the probability of discovering a target in a specific length of time. Thus relating accuracy to time (or equivalently speed) is of central concern, especially in instances where the respective goals are in conflict (e.g., safety and productivity). Moreover, search performance has been observed to vary markedly, in part because of distinct search behaviors and other individual differences (e.g., Wang, Lin, \& Drury, 1997). Hence there is also a need to establish performance benchmarks that represent the limits of search performance.

Search behavior, in particular, is commonly assumed to be influenced by both memory retrieval (e.g., Arani, Karwan, \& Drury, 1984) and

Address correspondence to Anand K. Gramopadhye, Clemson University, College of Engineering and Science, Advanced Technology Systems Laboratory, Clemson, SC 29634; agramop@ clemson.edu. HUMAN FACTORS, Vol. 48, No. 3, Fall 2006, pp. 540-554. Copyright (C) 2006, Human Factors and Ergonomics Society. All rights reserved 
search strategy (e.g., Williams, 1966). One aspect of search strategy is the degree of visual lobe overlap. The visual lobe, or visual field, is commonly defined as the area visible in a single fixation. Models have been developed that account for this overlap explicitly (Baveja, Drury, Karwan, \& Malon, 1996; Courtney \& Guan, 1996, 1998; Sarac, Batta, \& Drury, 1997) and implicitly (e.g., Arani et al., 1984; Drury \& Chi, 1995; Karwan, Morawski, \& Drury, 1995; Krendel \& Wodinsky, 1960; Lin, 1991; Morawski, Drury, \& Karwan, 1980, 1992; Williams, 1966), both of which have been validated in practice (e.g., Baveja et al., 1996, and Courtney \& Guan, 1998, in the former case and Drury \& Chi, 1995, Krendel \& Wodinsky, 1960, Morawski et al., 1980, and Williams, 1966, in the latter). The latter approach will be considered here.

The boundaries of search performance have previously been established using models based on diametric assumptions regarding search behavior. These two extreme cases are commonly referred to as systematic and random search. The former is characterized by systematic fixations and the latter by random fixations, as their names imply; these are analogous to sampling without and with replacement, respectively. Naturally, actual search behavior appears between these extremes.

Accordingly, Arani et al. (1984) developed a variable-memory simulation model to represent a search that is intended to be systematic but suffers from imperfect memory. (A mathematical model was derived as well, but it is tractable only under a very restrictive set of assumptions, thus motivating the development of the simulation.) The model incorporates a standard two-parameter decay/interference function memory model, which the authors stated could be estimated from eye movement data in practice. (In certain models [Courtney \& Guan, 1996, 1998] wherein lobe overlap is modeled explicitly, the degree of overlap characterizes the extent of memory loss. Hence from a modeling standpoint, it is arguable that the converse would be true in cases in which memory is modeled explicitly.)

A mathematical model for semisystematic search is proposed here that can account (primarily in an implicit manner) for memory retrieval and errors therein, and for other factors that could potentially affect performance. The latter may include participant factors such as motivation (e.g.,
Wiener, 1975); attitude toward risk (e.g., Megaw \& Richardson, 1979); individual differences in search strategy (e.g., Wang et al., 1997); specialized search strategies such as left-to-right, line-by-line patterns (e.g., Baveja et al., 1996); environmental factors such as noise (which could have either a favorable or an unfavorable effect on performance; e.g., Warner \& Heimstra, 1972); and temporal factors such as arousal (e.g., Poulton, 1973). Organizational factors such as training could also play a role, insofar as training would affect search strategy, for example (e.g., Gramopadhye, Drury, \& Prabhu, 1997).

At the core of the model is a function that characterizes search behavior over time. This function is not restricted to any particular form; as a result, it may better serve to parallel actual performance. The function can be estimated from accuracy (or other performance) data, which would be easier to obtain in practice than eye movement data. Moreover, the value of this function at a particular point in time corresponds to the systematic efficiency of the searcher at that juncture, thus providing a useful measure of individual performance. Finally, because a mathematical model is employed in lieu of a simulation, relationships between variables are more transparent and certain statistical problems inherent to simulations can be avoided.

\section{MODEL DEFINITION}

The process of searching a field for targets is modeled as a series of fixations. The search field itself is assumed to be homogeneous; that is, there are no regions that are distinctive, visually or otherwise. (Textiles, glass, sheet metal, castings, roller bearings, and lap-splice joints of fuselage structures are examples of homogeneous search fields, provided that the targets are inconspicuous.) It is represented as a set of equal-sized cells, with the size of these cells corresponding to the area that can be encompassed in a single fixation (commonly referred to as a hard-shell visual lobe). Each successive fixation either deliberately glimpses a cell not yet fixated in a systematic manner or arbitrarily glimpses a cell (which may or may not have been previously fixated) in a random fashion.

In order for a particular target to be located, two events must occur in succession: A cell containing a target must be fixated and the target subsequently perceived. It is assumed that the targets are 
inconspicuous, which precludes the possibility of a guided search (e.g., Wolfe, 1994). Thus, the likelihood of fixating on a particular cell containing a target is directly related to the number of fixations (which is directly proportional to the time engaged in search), relative to the size of the search field, for any established search behavior. It is further assumed that the targets are uniformly distributed over the search field and that a cell may contain at most one target. (In cases where the ratio of the number of cells to the number of targets is large, the probability that a single cell contains more than one target is negligible; Morawski et al., 1980.) Once a target is perceived, the search terminates.

However, it is not certain that a target will be perceived, even though the cell containing the target has been fixated. This uncertainty is attributable to factors such as the conspicuity of the target and its distance from the center of fixation. The conditional probability that a particular target is perceived, provided that the cell containing the target has been fixated on, will be referred to as the perceptual sensitivity. (The value of the perceptual sensitivity is inversely related to the size of the hard-shell visual lobe.) There may be several such probabilities, as the values usually differ according to the type of target. However, the probabilities do not vary with the location of a target, given that the search field is homogeneous. Lastly, the conditions stated are consistent with those of both Morawski et al. (1980) and Arani et al. (1984).

The model is intended to represent a semisystematic search that terminates upon detection of any target. Several parameters characterize the search:

$a$ : area of search field, $a \in \Re, \ni a>0$,

$o$ : visual lobe; that is, area of an individual cell, $o \in \Re, \ni o>0$,

$b_{j}$ : number of type $j$ targets in search field, $j=$ $1,2, \ldots, h, b_{j} \in Z+$

$g$ : search time limit (in seconds), $g \in \Re, \ni g>$ 0 ,

$h$ : number of different target types in search field, $h \in Z+$,

$\varepsilon_{t}$ : systematic search efficiency - that is, the probability of a systematic fixation at time $t$, $\varepsilon_{t} \in[0,1], t \in Z^{*}$, and

$\rho_{j}$ : perceptual sensitivity - that is, the proportion of time that a type $j$ target is perceived, given that the cell that contains it has been fixated, $\rho_{j} \in[0,1], j=1,2, \ldots, h$.

These parameters may also be considered in the context of performance shaping factors. For example, $a, b_{j}, g$, and $h$ are task factors, whereas $o$ is a participant factor. The parameter $\rho_{j}$ is affected both by task factors such as target conspicuity and by participant factors such as visual acuity. Lastly, $\varepsilon_{t}$ is the functional parameter that characterizes search behavior referred to in the previous section. Recall that its precise functional form will be defined by memory retrieval and various other performance-shaping factors, such as individual differences in search strategy. Thus the model can implicitly incorporate the forenamed stationary and nonstationary (temporal) factors, as well as others, via this function.

\section{MODEL FORMULATION}

The fundamental modeling approach is based on the concept of what will be referred to here as a scan, the number of distinct cells fixated within a particular scan, the numbers and types of targets contained in the partition formed by these cells, and whether or not one of the targets in this partition is perceived. The concepts of scan and distinct cells will be clarified before continuing. A scan is essentially a measure of coverage that segments the search into blocks of $n$ distinct fixations, in which $n$ corresponds to the number of cells in the search field. The latter is established by computing the ratio of the area of the entire field to that of the visual lobe:

$$
n=\lceil a / o\rceil .
$$

A fixation is considered to be distinct if the newly fixated cell has not already been glimpsed during the current scan. Once $n$ distinct fixations have occurred, the current scan is complete; the next fixation demarcates a new scan. In other words, once a new scan begins, the slate is wiped clean, so to speak, and all new fixations are considered distinct until one of the cells in this new partition is refixated.

Adopting this approach, the search process will be modeled as a discrete-time nonstationary Markov process (e.g., Ross, 2003). The states of the process, $X_{t}$, will be represented by a 3 -tuple 
$(k, \mathbf{l}, m)$. The substance of these three indexes was alluded to previously. Descriptions of the individual indexes and the relationships that exist between them will now be presented by considering the search as it progresses from the outset, through the completion of the first scan, to the initiation of the second.

The initial scan commences as the search is initiated, with a series of fixations. The first index, $k$, is a nonnegative integer, the value of which corresponds to the number of distinct cells that have been fixated. It is therefore a measure of field coverage, which of course cannot exceed the cumulative number of fixations. The maximum value of $k$ corresponds to the maximum number of fixations, $f$, which is the quotient of

$$
f=\lfloor g / 0.3\rfloor,
$$

in which $0.3 \mathrm{~s}$ is the duration of a single fixation (e.g., Arani et al., 1984). (The number of fixations is often used herein to express the concept of time.) Each successive fixation will either increment $k$ by 1 if the newly fixated cell is distinct or leave $k$ unchanged if it is not. Hence $k$ is a nondecreasing variable.

The second index, $\mathbf{l}$, is a vector that has $h$ elements, each of which corresponds to a different target type. The $j$ th element, $l_{j}$, is a nonnegative integer with a maximum value of $b_{j}$, in which $b_{j}$ denotes the number of type $j$ targets in the search field. (Herein, the convention will be to represent all vectors in row form.) These elements serve to enumerate the various types of targets that are contained in the partition formed by the cells that have been fixated. It follows therefore that

$$
l_{\Sigma} \leq k
$$

for $k=0,1, \ldots, f$, in which

$$
l_{\Sigma}=\sum_{j=1}^{h} l_{j}
$$

for $l_{j}=0,1, \ldots, b_{j}$. Similarly,

$$
n-k \geq b_{\Sigma}-l_{\Sigma},
$$

in which

$$
b_{\Sigma}=\sum_{j=1}^{h} b_{j} \text {, }
$$

or equivalently,

$$
k \leq n-\left(b_{\Sigma}-l_{\Sigma}\right),
$$

because $(n-k)$ and $\left(b_{\Sigma}-l_{\Sigma}\right)$ represent the number of cells and targets that have not yet been fixated, respectively.

On each successive fixation (with the exception of the fixation that initiates a new scan), either one of the elements will be incremented by 1 if the newly fixated cell is distinct and it contains a target, or else, if not, the elements will remain unchanged. In the former case, this index will be expressed as $\mathbf{I}+\mathbf{I}_{\mathbf{j}}^{\prime}$, in which $\mathbf{I}_{\mathbf{j}}^{\prime}$ designates the transpose of the $j$ th column of the identity matrix (or, i.e., its $j$ th row, in accordance with the convention of representing all vectors in row form), indicating that the distinct cell fixated contains a type $j$ target. Once all of the targets have been fixated, $l_{j}=b_{j}$ for $j=1,2, \ldots, h$, or equivalently, $\mathbf{l}=\mathbf{b}$.

The third index, $m$, is a binary variable that indicates whether or not a fixated target has been perceived; that is,

$$
m= \begin{cases}0 & \begin{array}{l}
\text { when target is not perceived } \\
\text { on } t \text { th fixation, or }
\end{array} \\
1 & \begin{array}{l}
\text { when target is (fixated and) } \\
\text { perceived on } t \text { th fixation }
\end{array}\end{cases}
$$

Clearly,

$$
m \leq l_{\Sigma}
$$

Recall that the search terminates whenever a target is perceived.

Once all cells in the field have been fixated (at least once), a scan is considered to be complete; hence at this stage (the end of the first scan), $k=$ $n$. Moreover, because all of the targets must have been fixated, it follows that $\mathbf{l}=\mathbf{b}$. Reaching this stage signifies that a target has not been perceived on the previous $(t-1)$ fixations. If a target is perceived on the $t$ th fixation, then $m=1$ and the search is terminated. Otherwise, the next fixation demarcates a new scan, whereupon $\mathbf{l}$ will be reinitialized. After this fixation, either $\mathbf{l}=\mathbf{0}$, indicating that the cell fixated does not contain a target, or $\mathbf{l}=\mathbf{I}_{\mathbf{j}}^{\prime}$, indicating that this cell contains a type $j$ target. The index $k$ is not reinitialized, however, as it is a cumulative measure of coverage. Instead, a function, $r_{k}$, is created, 


$$
r_{k}= \begin{cases}k(\bmod n) & k=0,1, \ldots, f, \ni k \neq n, 2 n, \ldots \\ n & k=n, 2 n, \ldots\end{cases}
$$

so that its value corresponds to the number of distinct cells that have been fixated during the current scan. Thus, in general, $r_{k}=n$ at the end of the $i$ th scan, $i \in Z+$, and

$$
m \leq l_{\Sigma} \leq r_{k} \leq n-\left(b_{\Sigma}-l_{\Sigma}\right),
$$

because of Equations 3, 4, 6, 7, 9, and 10. (It will be seen that this function also plays a central role in determining the transition probabilities.) Finally, this description (and the specifics) would apply to subsequent scans without loss of generality, other than $k=n$ at the end of no scan other than the first.

Thus, the states of the Markov process may now be represented as

$$
X_{t}=(k, \mathbf{l}, m)
$$

for $(k, \mathbf{l}, m) \in \Theta_{t}$, in which $\Theta_{t}$ is the indexed set of states $(k, \mathbf{l}, m)$ for all $k, \mathbf{l}$, and $m$ such that $m \leq l_{\Sigma} \leq$ $r_{k} \leq n-\left(b_{\Sigma}-l_{\Sigma}\right)$ and $k \leq t$, for $t=0,1, \ldots, f$, because of Equations 4, 6, 10, and 11. The transitions of the process form three distinct sets:

$$
\begin{aligned}
& (k, \mathbf{l}, 0) \rightarrow(k+1, \mathbf{l}, 0),\left(k+1, \mathbf{l}+\mathbf{I}_{\mathbf{j}}^{\prime}, 0\right),(k+1, \mathbf{l}+ \\
& \left.\mathbf{I}_{\mathbf{j}}^{\prime}, 1\right),(k, \mathbf{l}, 0),(k, \mathbf{l}, 1) \text { for } k \neq n, 2 n, \ldots, \\
& (k, \mathbf{b}, 0) \rightarrow(k+1, \mathbf{0}, 0),\left(k+1, \mathbf{I}_{\mathbf{j}}^{\prime}, 0\right),\left(k+1, \mathbf{I}_{\mathbf{j}}^{\prime}, 1\right) \\
& \text { for } k=n, 2 n, \ldots,\left[\frac{f-1}{n}\right], \text { and } \\
& (k, \mathbf{l}, 1) \rightarrow(k, \mathbf{l}, 1) .
\end{aligned}
$$

The first set contains transitions that occur during an ongoing search at any time other than when a scan is completed, the second set includes those transitions that occur only at the time a scan is completed, and the transitions in the third set indicate that a target has been perceived and the search terminated.

The conditions that define the transitions within these sets will now be described. To begin, the transition $(k, \mathbf{l}, 0) \rightarrow(k+1, \mathbf{l}, 0)$ will be considered. First observe that a distinct cell has been fixated, given that the first index of the destination 3-tuple has a value of $(k+1)$. Such a state change may oc- cur by means of either a random or a systematic fixation. Moreover, because the middle index remains unchanged, this implies that the new cell fixated does not contain a target. As a result, the third index necessarily has a value of 0 because a target (that is not present) cannot be perceived. Next, the transitions $(k, \mathbf{l}, 0) \rightarrow\left(k+1, \mathbf{l}+\mathbf{I}_{\mathbf{j}}^{\prime}, 0\right)$ and $(k, \mathbf{l}, 0) \rightarrow\left(k+1, \mathbf{l}+\mathbf{I}_{\mathbf{j}}^{\prime}, 1\right)$ differ from the previous one in the respect that the distinct cell fixated contains a type $j$ target, because the $j$ th element of the middle index of the destination 3-tuple has been incremented by one. In the former case the target is not perceived, whereas in the latter case it is, as indicated by the respective values of the third index of the destination 3-tuple.

Conversely, the transitions $(k, \mathbf{l}, 0) \rightarrow(k, \mathbf{l}, 0)$ and $(k, \mathbf{l}, 0) \rightarrow(k, \mathbf{l}, 1)$ signify instances in which a cell is refixated, because the first index is unchanged. Thus these particular transitions must be the result of a random fixation. The second index necessarily remains the same because the partition (of distinct cells) has not been expanded to encompass additional targets. In the former case, a target is not perceived; this may be attributable either to a failure to perceive a target when the cell that contains it is refixated or to simply refixating a cell that does not contain a target. However, the latter transition reflects an instance in which the refixated cell includes a target that is perceived.

In contrast to the first set of state transitions described previously, those included in the second set, $(k, \mathbf{b}, 0) \rightarrow(k+1, \mathbf{0}, 0),(k, \mathbf{b}, 0) \rightarrow\left(k+1, \mathbf{I}_{\mathbf{j}}^{\prime}, 0\right)$ and $(k, \mathbf{b}, 0) \rightarrow\left(k+1, \mathbf{I}_{\mathbf{j}}^{\prime}, 1\right)$, occur only at the instant a scan is completed. In this case, the second index of the origination 3-tuple must equal $\mathbf{b}$ because the partition envelops the entire field, and thus all the targets, once a scan is completed. Accordingly, this index is reinitialized in the destination 3tuples, because the commencement of a new scan creates a new partition. Similarly, the first index is incremented because the first fixation of a new partition is necessarily distinct. The specific realizations of the second and third indices (of the destination 3-tuples) of this set of transitions are interpreted in a manner identical to that of the previous set. The last transition to be considered, $(k, \mathbf{l}, 1) \rightarrow(k, \mathbf{l}, 1)$, is characteristic of an absorbing state (in a Markov process). In the current context, absorption occurs when a target is perceived, because the search is terminated at that point.

Finally, the likelihood of any particular state 
change is governed by a set of transition probabilities. These probabilities are obtained by considering the conjunction of several events. For example, consider the transition $(k, \mathbf{l}, 0) \rightarrow(k+$ $1, \mathbf{l}, 0)$ once again. Recall that this transition may occur via either a random or systematic fixation. Under the assumption of the former, four events must occur: There will first be a random fixation; this fixation will glimpse a distinct cell; the fixated cell will not include a target; and a target will not be perceived. A systematic fixation, of course, alters the first event but not the others. Because random and systematic fixations are exclusive, the respective probabilities of these events would be added. In this manner, the equation for the probability of this particular transition, denoted by $p_{(k, \mathbf{1}, 0),(k+1, \mathbf{l}, 0)}^{t}$, is obtained:

$$
\begin{gathered}
p_{(k, 1,0),(k+1,1,0)}^{t}=\left(1-\varepsilon_{t}\right) \cdot\left(\frac{n-r_{k}}{n}\right) . \\
\left(\frac{n-r_{k}-\left(b_{\Sigma}-l_{\Sigma}\right)}{n-r_{k}}\right) \cdot 1+\varepsilon_{t} \cdot 1 . \\
\left(\frac{n-r_{k}-\left(b_{\Sigma}-l_{\Sigma}\right)}{n-r_{k}}\right) \cdot 1= \\
\left(n-r_{k}-b_{\Sigma}+l_{\Sigma}\right)\left(\frac{1-\varepsilon_{t}}{n}+\frac{\varepsilon_{t}}{n-r_{k}}\right) .
\end{gathered}
$$

The other transition probabilities are derived in a similar fashion. A complete set of transition probabilities may be found in the Appendix. It is noteworthy that models for both random and systematic searches could be obtained by setting $\varepsilon_{t}=0$ and $\varepsilon_{t}=1$ in these equations, respectively, for all $t$.

\section{PERFORMANCE MEASURES}

There are several measures of interest, one of which, the mean systematic search efficiency, $\bar{\varepsilon}$, can be determined directly from averaging the systematic search efficiency at each time epoch:

$$
\bar{\varepsilon}=\frac{1}{f} \sum_{t=0}^{f-1} \varepsilon_{t} .
$$

The others,

$\alpha_{t}$, accuracy - (cumulative) probability of perceiving a target by time $t, \alpha_{t} \in[0,1]$;

$V$, time to perception - number of fixations required to perceive a target, $v=1,2, \ldots f$;

$W$, task time - number of fixations expended in search task effort, irrespective of whether or not a target is perceived, $w=1,2, \ldots f$; and

$C_{t}$, coverage-number of distinct cells fixated by time $t$ in the initial scan of a field void of targets, relative to the number of cells in the field, $C_{t} \in(0,1]$;

are output measures in the strictest sense. These metrics are a function of the transition probabilities; they are also a function of state probabilities.

Let $q_{(k, \mathbf{l}, m)}^{t}$ represent the probability that state $(k, \mathbf{l}, m)$ is occupied at time $t$; that is,

$$
q_{(k, \mathbf{l}, m)}^{t}=\operatorname{Pr}\left[X_{t}=(k, \mathbf{l}, m)\right],
$$

for $(k, \mathbf{l}, m) \in \Theta_{t}$, for all $t$. Now let $q_{(k, \mathbf{l}, m)}^{t}$ be the $(k, \mathbf{l}, m)$ th element of the state probability vector $\mathbf{q}^{\mathbf{t}}$. Also let $p_{(k, \mathbf{l}, 0),(k+1, \mathbf{l}, 0)}^{t}$ be the $(k, \mathbf{l}, 0)(k+1, \mathbf{l}, 0)$ th element of the transition probability matrix $\mathbf{P}^{\mathbf{t}}$, let $p_{(k, \mathbf{l}, 0),\left(k+1, \mathbf{l}+\mathbf{I}_{\mathbf{j}, 0)}\right)}^{t}$ be the $(k, \mathbf{l}, 0)\left(k+1, \mathbf{l}+\mathbf{I}_{\mathbf{j}}^{\prime}, 0\right)$ th element of $\mathbf{P}^{\mathbf{t}}$, and so on. Then

$$
\mathbf{q}^{\mathbf{t}}=\mathbf{q}^{\mathbf{t}-\mathbf{1}} \mathbf{P}^{\mathbf{t}-\mathbf{1}},
$$

for $t=1,2, \ldots, f$, with

$$
\mathbf{q}^{0}=[1,0,0, \ldots, 0],
$$

because $q_{(0,0,0)}^{0}=1$.

Now, the accuracy at time $t$ is expressed in terms of the absorbing state probabilities as

$$
\alpha_{t}=\sum_{k} \sum_{\substack{1 \\(\mathrm{k}, \mathbf{l}, 1) \in \Theta_{t}}} q_{(k, \mathbf{l}, 1)}^{t}
$$

for $t=1,2, \ldots, f$, because absorption and target perception are synonymous in this context.

The mean has been selected to characterize the remaining measures, as they are random variables. The equation for the second measure, the expected time to perception, is

$$
\mu_{V}=\sum_{v=1}^{f} v d_{V}(v),
$$

in which $d_{V}(v)$ denotes the mass function for the random variable. The equation for the mass function

$$
d_{V}(v)=\operatorname{Pr}(V=v)=D_{V}(v)-D_{V}(v-1),
$$


for $v=1,2, \ldots, f$, can be readily found using the distribution function, $D_{V}(t)$, in which

$$
D_{V}(t)=\operatorname{Pr}(V \leq t)=\frac{\alpha_{t}}{\alpha_{f}}
$$

for $t=1,2, \ldots, f$, because the absorbing state probabilities are cumulative over time.

Next, recall that the search will terminate in one of two ways: when either a target is perceived or time has lapsed as the consequence of an unsuccessful search. Hence, the expression for the expected task time is a convex combination of the mean time to perception and the time limit, weighted by the respective probabilities of a "hit" and a "miss":

$$
\mu_{W}=\alpha_{f} \mu_{V}+\left(1-\alpha_{f}\right) f
$$

The final measure is derived from a field void of targets. Hence in this particular case $\mathbf{l}=\mathbf{b}=\mathbf{0}$, from which it follows that

$$
\sum_{k=1}^{t} q_{(k, \mathbf{0}, 0)}^{t}=1
$$

for $t=1,2, \ldots, f$. The resultant equation for the expected coverage at time $t$, then, is

$$
E\left[C_{t}\right]= \begin{cases}\frac{1}{n} \sum_{k=1}^{t} k q_{(k, \mathbf{0}, 0)}^{t}, & t \leq n \\ \frac{1}{n} \sum_{k=1}^{n} k q_{(k, \mathbf{0}, 0)}^{t}+\sum_{k=n+1}^{t} q_{(k, \mathbf{0}, 0)}^{t}, & t>n\end{cases}
$$

for $t=1,2, \ldots, f$.

Heretofore, it has been assumed that the numbers of the various types of targets present (in the field) are known with certainty. Indeed, this would be the case in a synthetic task environment in the context of training, for example. However, if the numbers are not known (with certainty), then $\mathbf{b}$ would not be fixed but instead would represent a realization of a random vector, $\mathbf{B}$. Nonetheless, all of the output measures could still be found by using a theorem of conditional expectation. For example, the equation for the expected value of the task time would become
$\mu_{W}=E[E[W \mid \mathbf{B}]]=\sum_{\mathbf{b}} E[W \mid \mathbf{B}=\mathbf{b}] \operatorname{Pr}(\mathbf{B}=\mathbf{b})$,

in which $E[W \mid \mathbf{B}=\mathbf{b}]$ would be given by Equation 22 . These performance measures will now be considered via a numerical example.

\section{MODEL ILLUSTRATION}

An example will be adapted from Arani et al. (1984) in which $n=50, f=200$ (i.e., $g=60$ s), $h=$ 2, $\operatorname{Pr}(\mathbf{B}=[1,1])=\operatorname{Pr}(\mathbf{B}=[1,2])=\operatorname{Pr}(\mathbf{B}=[2,1])=$ $\operatorname{Pr}(\mathbf{B}=[2,2])=0.25$, and $\rho=[0.8,0.5]$. The corresponding state transition diagram is depicted in Figure 1. Now, for the current model, let the systematic search efficiency be subject to exponential decay; specifically, let $\varepsilon_{t}=x y^{\frac{t}{z}}, x \in(0,1], y \in$ $[0,1], z \in \Re, \ni z>0$, for $t=0,1, \ldots, f-1$. Although $x$ will be fixed at 1 and $z$ at 50 (that is, $n$ ) here, several values of $y$ will be considered in order to demonstrate how different rates of decay affect the various performance measures. These values are listed in Table 1, along with the corresponding average systematic efficiencies. In addition, plots of the different systematic efficiencies over time are depicted in Figure 2. The first value yields a random search (with the condition that $0^{0}=0$ ). Cases 2 through 4 produce searches that become random after 100, 150, and 200 fixations, respectively. Cases 5 through 9 generate searches that have respective systematic search efficiencies of $1 \%, 5 \%, 10 \%, 20 \%$, and $40 \%$ after 60 s. Of course, the last value yields a search that is strictly systematic.

Figure 3 reveals that coverage is directly related to the degree of systematic efficiency, as expected. Moreover, this figure confirms that in the case of strictly systematic search, the field coverage is equal to 1 (or $100 \%$ ) when the number of fixations corresponds to the field size. (This also coincides with the point at which the difference in coverage yielded by the extreme behaviors reaches a maximum.) The expected field coverage of the other cases will approach - but never achieve - a value of 1 (for any finite number of fixations), because complete coverage is not certain when any random behavior is exhibited. Next, observe the striking similarities between the expected coverage curves and the corresponding accuracy curves, shown in Figure 4. In particular, observe how closely the 

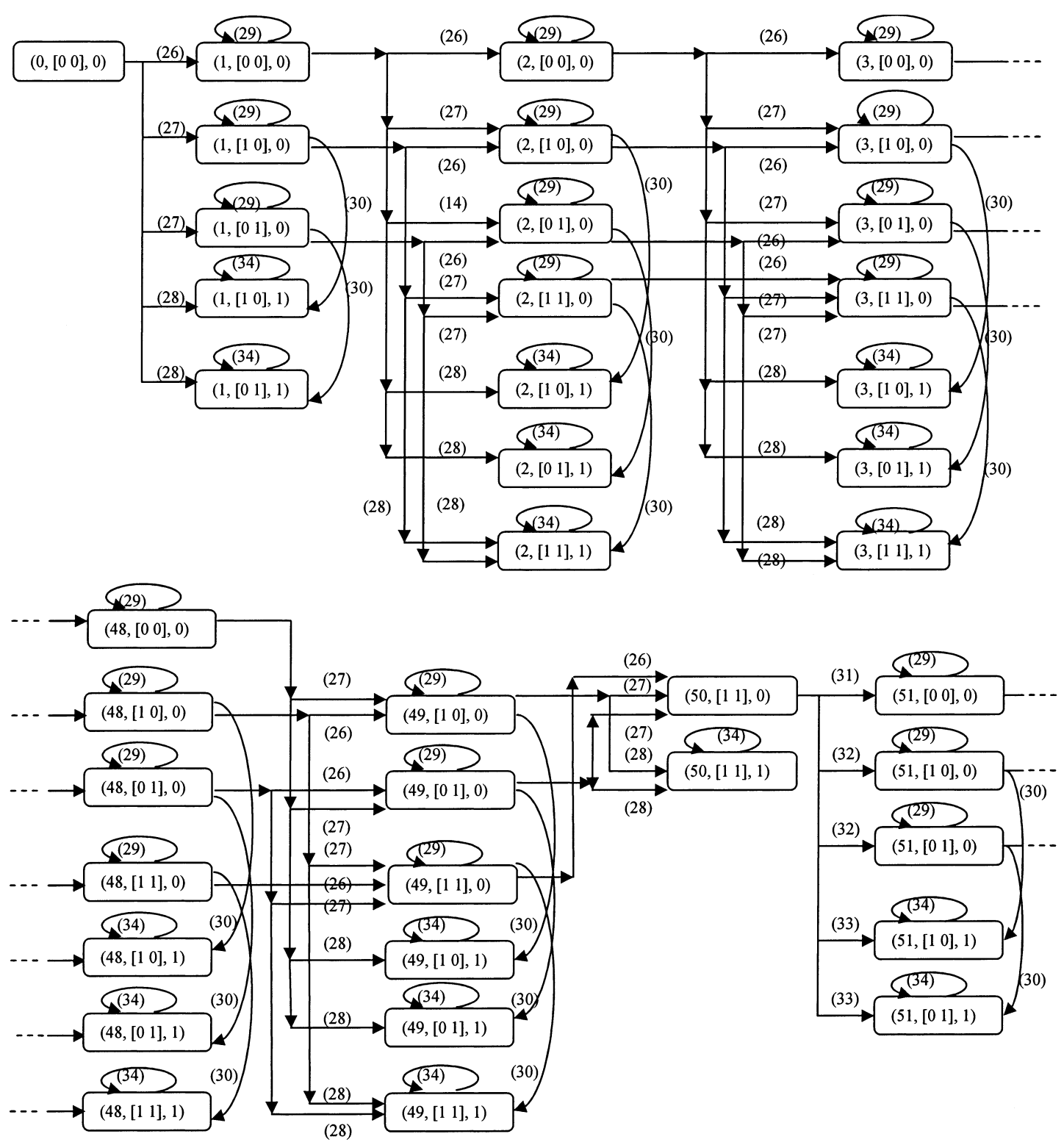

Figure 1. State transition diagram.

curves of Cases 2 and 3 parallel that of the random search, and that Cases 6 through 9 converge at 75 fixations. Thus these figures suggest a direct link between expected coverage and accuracy.

The expected perception times for the various cases, however, tend to diverge at first as the number of fixations increases, as displayed in Figure 5. Moreover, although the curves of the different cases maintain their respective positions with respect to accuracy and coverage, the perception time curves do not. The cases that are rel- atively less systematic exhibit perception times that are initially smaller, and later larger, than their counterparts. The reason for this is that when the less systematic searches are successful, it is more likely that they will be successful early on, as illustrated by the extreme cases in Figure 6a. This initial advantage is negated as the time horizon is extended, however, because protracted searches are more likely to be the by-product of less efficient behavior, as demonstrated in Figure $6 \mathrm{~b}$. Moreover, because it is less likely that 
TABLE 1: Selected Values of $y$ With Corresponding Average Systematic Search Efficiencies

\begin{tabular}{cll}
\hline Case & \multicolumn{1}{c}{$y$} & \multicolumn{1}{c}{$\bar{\varepsilon}$} \\
\hline 1 & .0 & .0 \\
2 & .028747 & .073 \\
3 & .076524 & .100 \\
4 & .171375 & .144 \\
5 & .318314 & .219 \\
6 & .473083 & .320 \\
7 & .562446 & .393 \\
8 & .668760 & .499 \\
9 & .795242 & .656 \\
10 & 1.0 & 1.0 \\
\hline
\end{tabular}

these searches will be successful, the expected task time curves (which represent a weighted combination of the perception and unsuccessful termination times) reflect the fact that less efficient searches are consistently more time consuming on average, as shown in Figure 7.

\section{CONCLUSION}

An extended semisystematic search was modeled with a discrete-time nonstationary Markov process. The time-dependent, semisystematic search behavior is expressed by an embedded function. Given its generic nature, this function is capable of generating not only time-dependent decreases in efficiency but increments as well, if appropriate. The function can be estimated from performance data such as accuracy, or process data such as coverage, although the latter are usually more difficult to obtain in practice.

The present model requires no assumptions beyond those applied by Morawski et al. (1980) to their models for random and strictly systematic search, despite its capacity to reproduce these behaviors, as well as those characteristic of semisystematic search. (These particular models are underscored because they are extensions of earlier models of random [e.g., Krendel \& Wodinsky, 1960] and strictly systematic search [e.g., Williams, 1966].) The same is also true with regard to the assumptions imposed in the variable-memory simulation model developed by Arani et al. (1984). Nevertheless, the mathematical model proposed here embodies both memory-related factors and other determinants, and it is not subject to the statistical difficulties intrinsic to simulation methods.

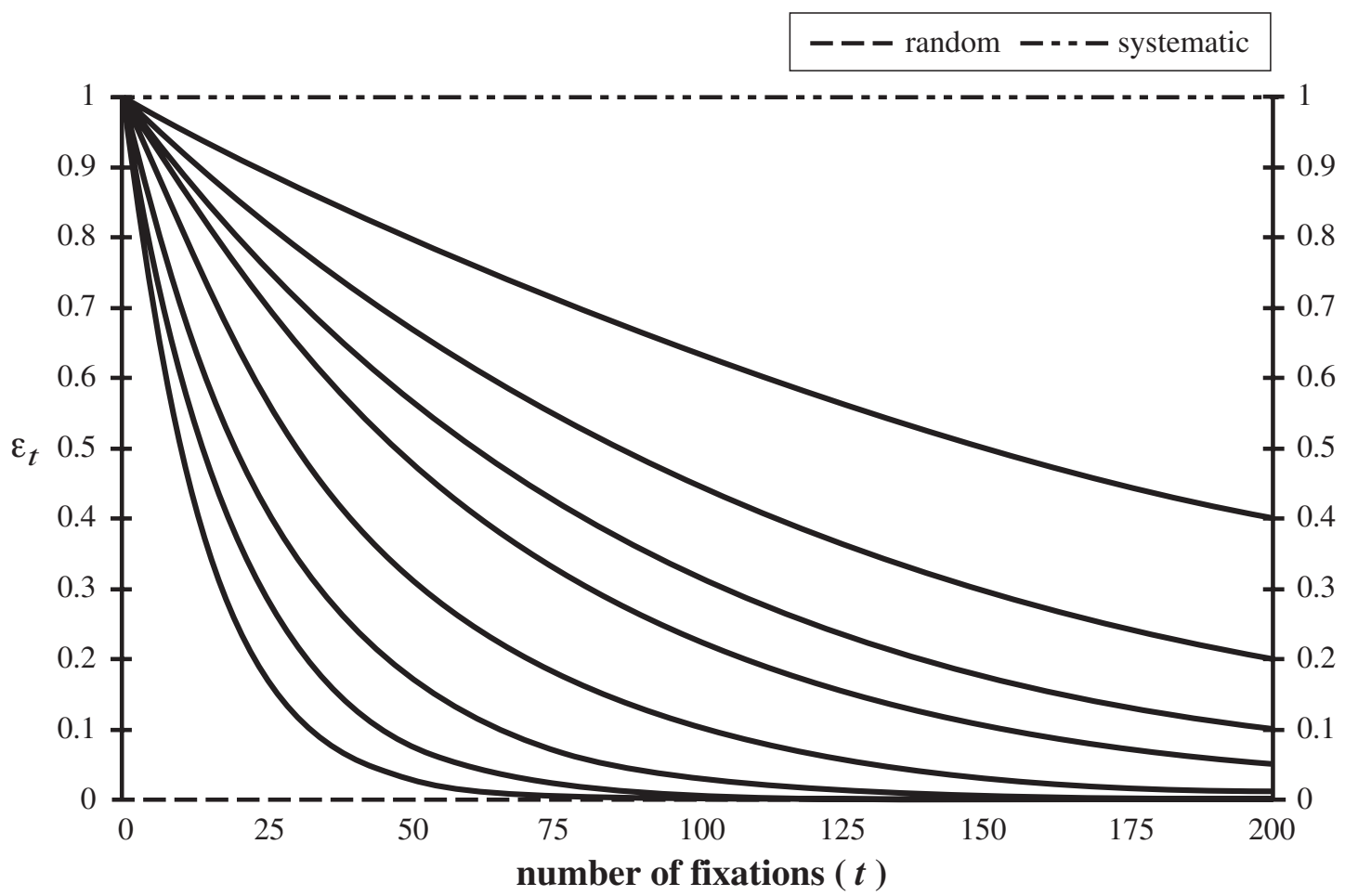

Figure 2. Systematic search efficiency versus number of fixations. 


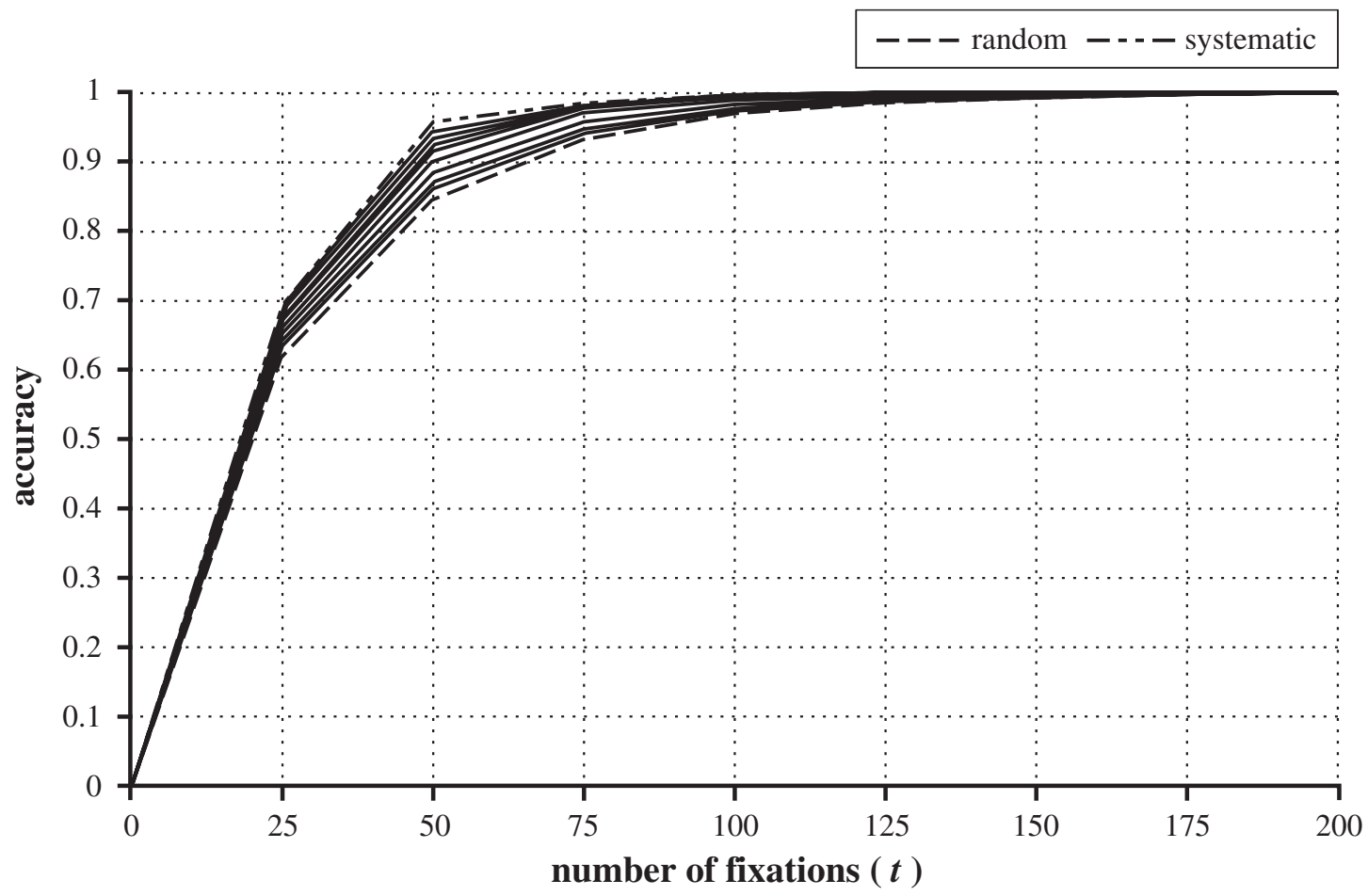

Figure 3. Accuracy versus number of fixations.

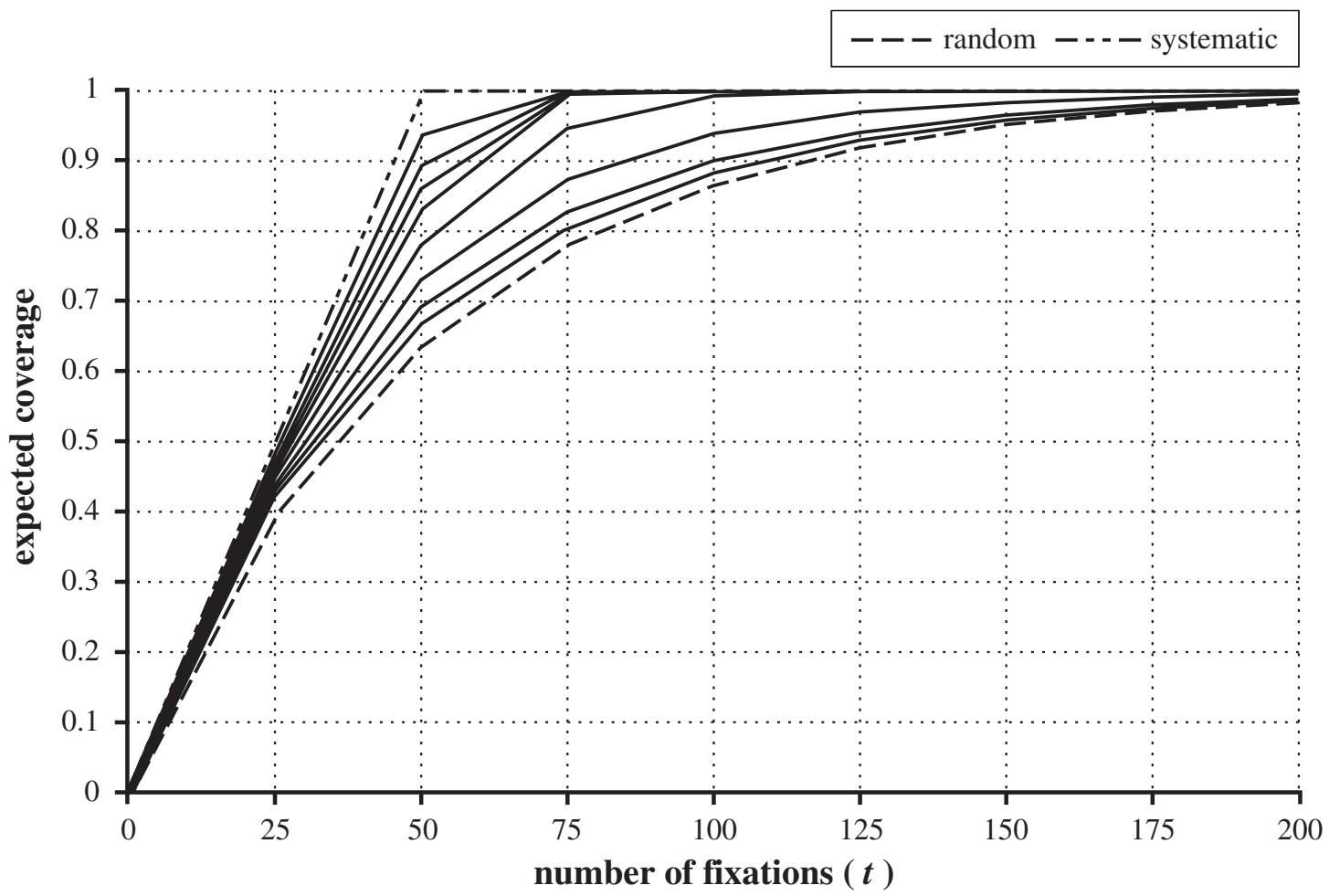

Figure 4. Expected empty field coverage versus number of fixations. 


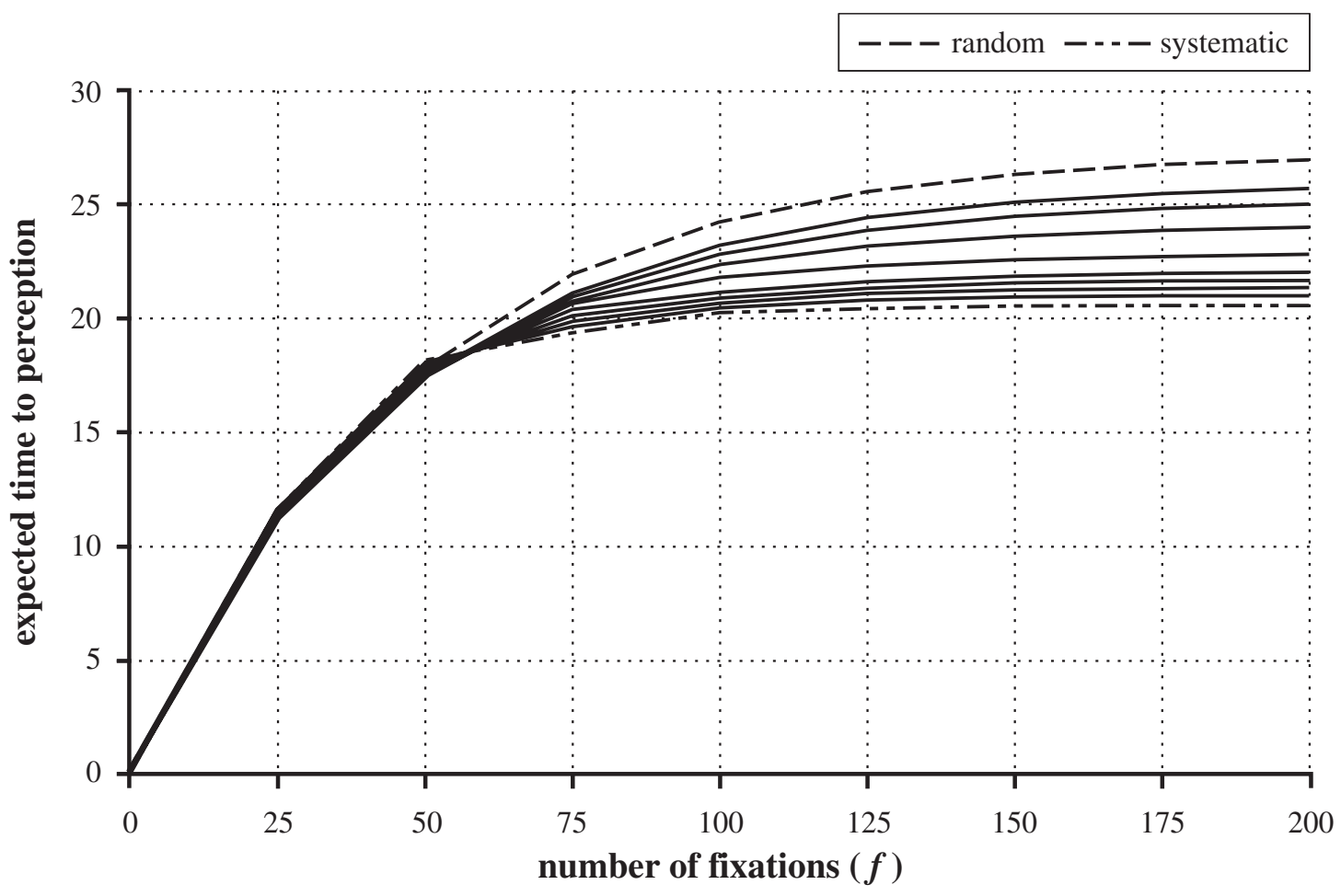

Figure 5. Expected time to perception versus maximum number of fixations.

Moreover, this model generates both performance and process measures, whereas the variable-memory model yields only accuracy. Although the other models yield mean and median times to perception, in addition to accuracy, these values represent approximations that are apparently based on an infinite time horizon. Consequently, a single value is produced, irrespective of the time limit.

Specifically, the measures that the present model is able to produce are accuracy, field coverage, time to perception, and task time. In particular, it was seen that as the search behavior becomes more systematic, expected coverage and accuracy increase and expected task time decreases. These outcomes are consistent with empirical studies (e.g., Megaw \& Richardson, 1979; Schoonard \& Gould, 1973; Wang et al., 1997) and hence support the validity of the model. It was also observed that whereas increasingly systematic behavior ultimately yields smaller expected perception times, the reverse is true initially, which represents a finding that is neither confirmed nor contradicted by the literature (to the best of our knowledge).
In addition to explaining these outcomes and their interrelationships from a theoretical standpoint, the model can predict these outcomes in practice to a certain extent as it can create an envelope defined by best- and worst-case search performances. The practical value of the model for predicting intermediate performance is arguable, however, because doing so would require the estimation of the search efficiency parameter by means of either a pilot study or past data from similar tasks. Nevertheless, it is noteworthy that the model also has the capability of supporting assessment. That is, it can be used to assess the effectiveness of an individual's search performance, and to provide possible explanations for this performance, through the use of one or more of the output measures. In this manner, the model can serve both initially to screen candidates for visual search tasks and, subsequently, to identify interventions for those who routinely perform these tasks. Finally, although the application of this model is currently confined to homogeneous search fields, it potentially could be adapted to incorporate heterogeneous regions. Such a model would not only have intrinsic value 


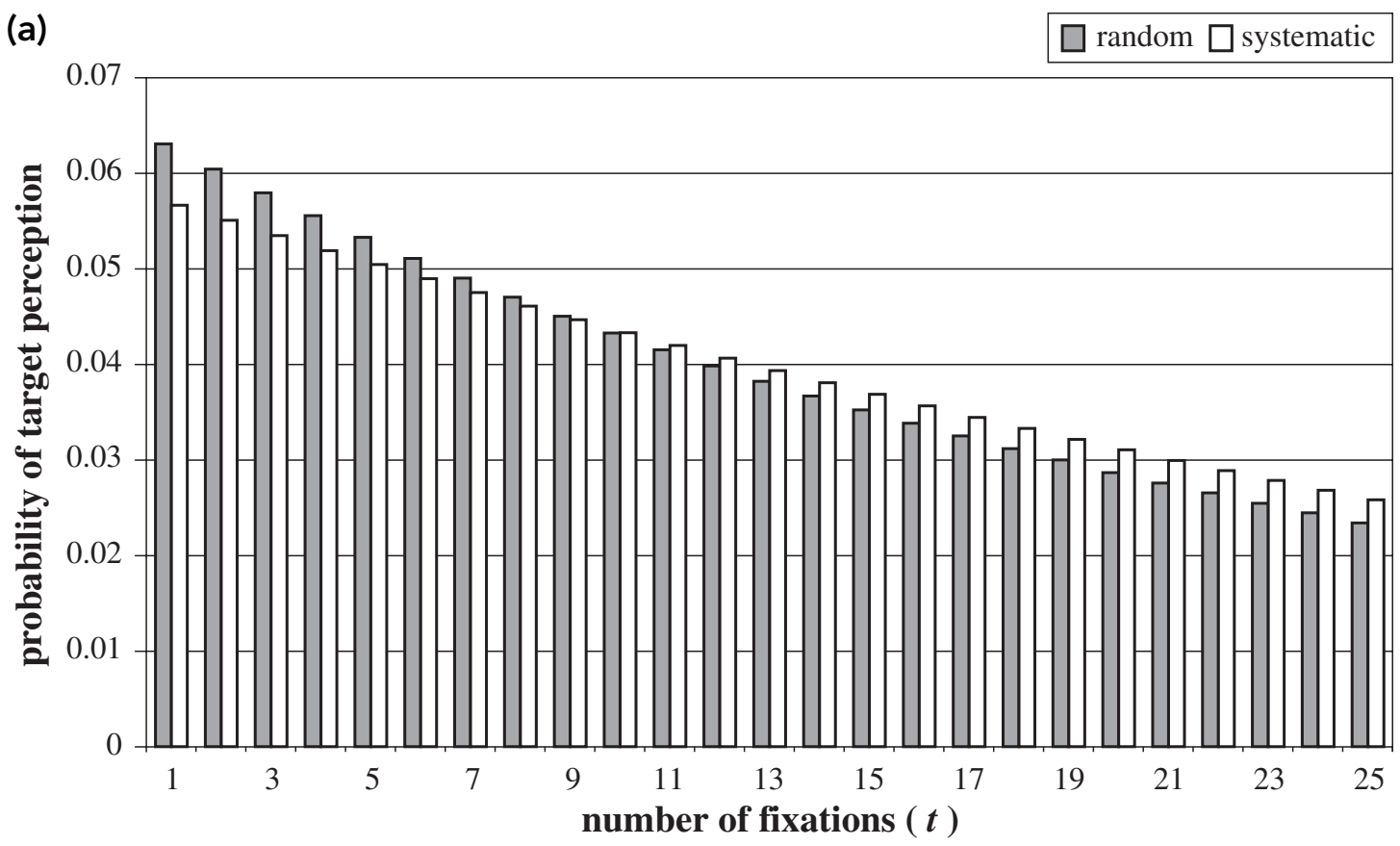

(b)

random systematic

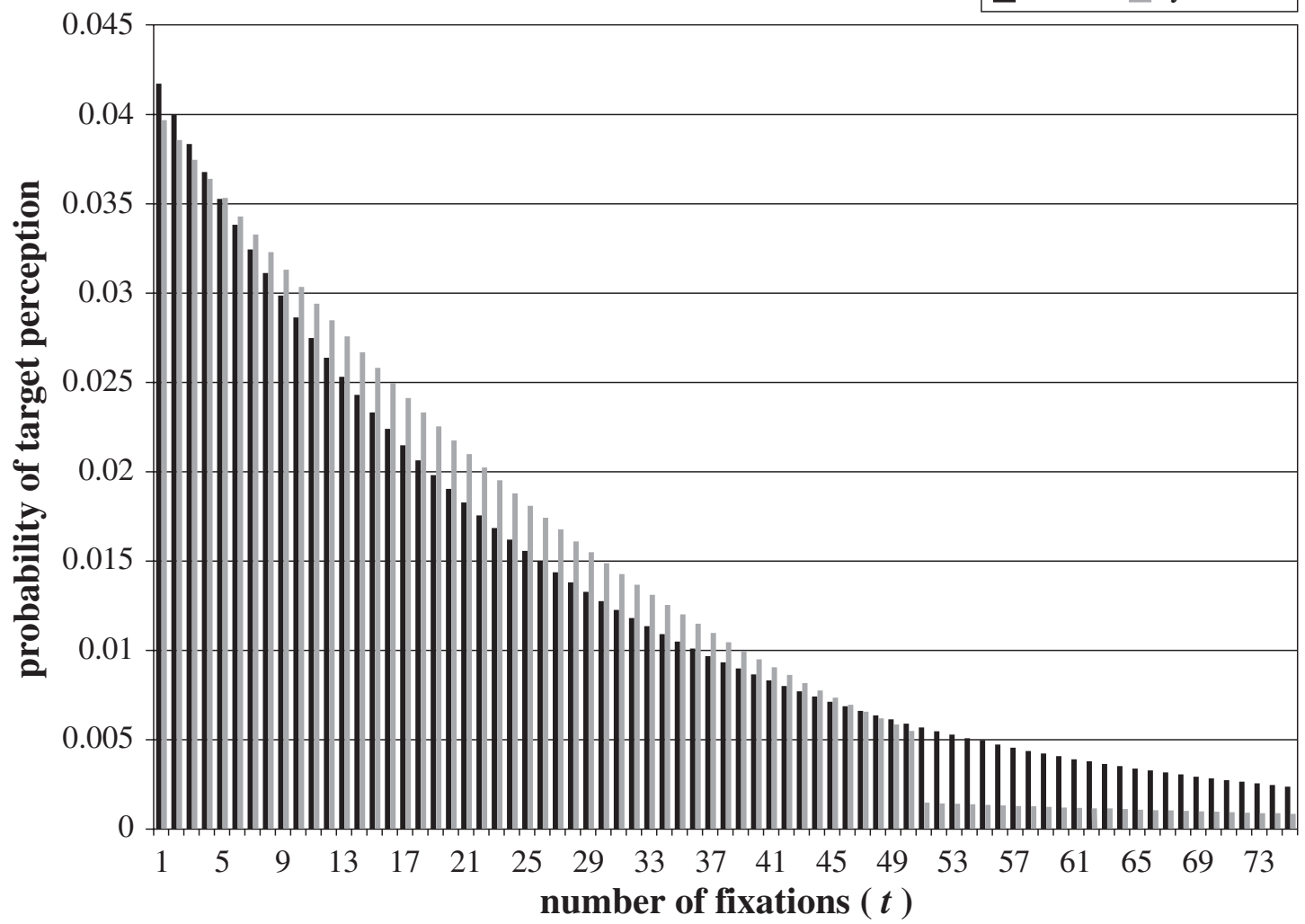

Figure 6. Probability of target perception versus number of fixations for random and strictly systematic searches over abbreviated (a, above) and expanded (b, below) horizons. 


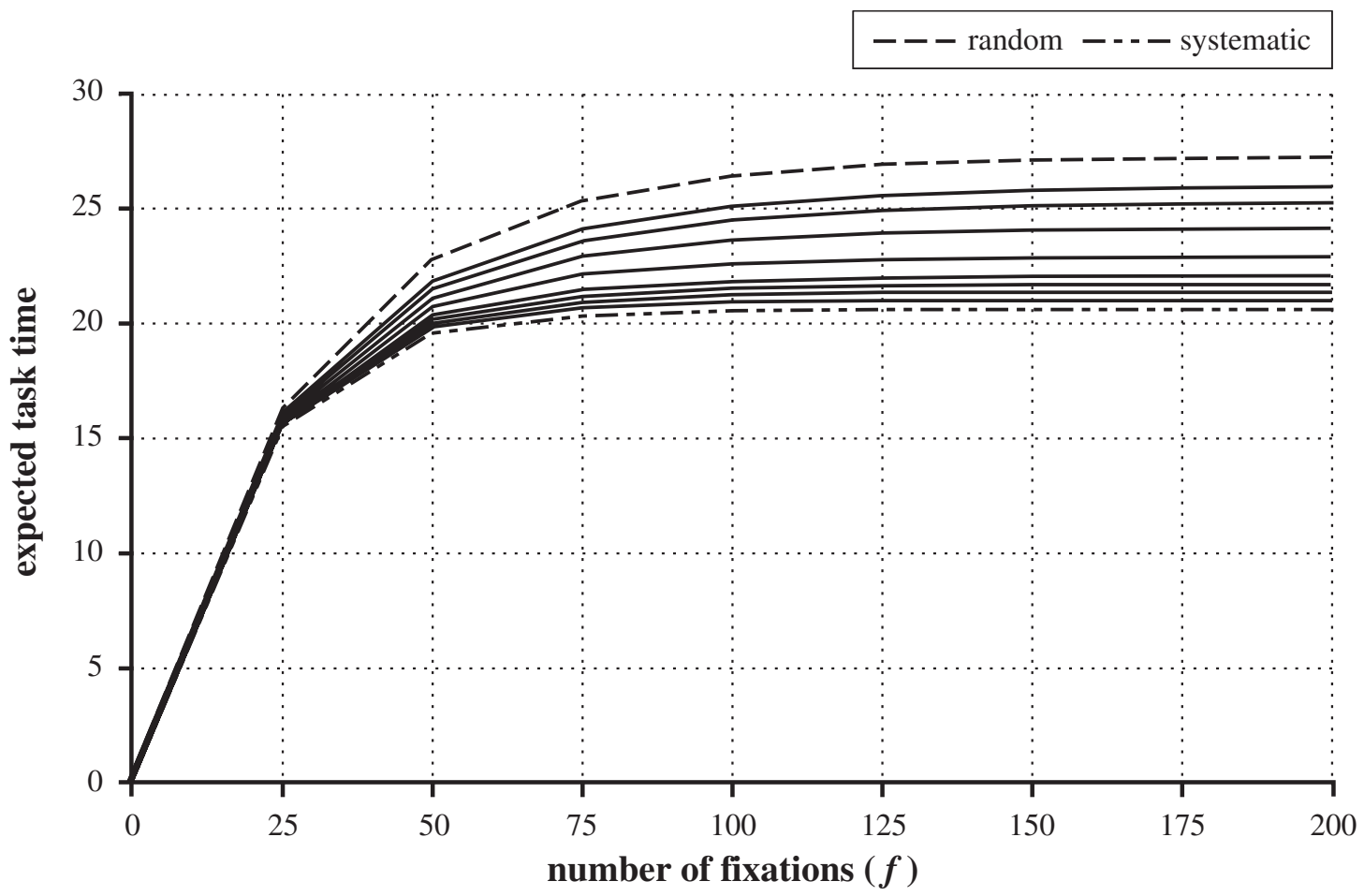

Figure 7. Expected task time versus maximum number of fixations.

but would also represent the next step in the prospective development of a model for extended guided search.

\section{APPENDIX}

Recall that the transitions of the process constitute three distinct sets:

$(k, \mathbf{l}, 0) \rightarrow(k+1, \mathbf{l}, 0),\left(k+1, \mathbf{l}+\mathbf{I}_{\mathbf{j}}^{\prime}, 0\right),(k+1, \mathbf{l}+$ $\left.\mathbf{I}_{\mathbf{j}}^{\prime}, 1\right),(k, \mathbf{l}, 0),(k, \mathbf{l}, 1)$,

$(k, \mathbf{b}, 0) \rightarrow(k+1, \mathbf{0}, 0),\left(k+1, \mathbf{I}_{\mathbf{j}}^{\prime}, 0\right),\left(k+1, \mathbf{I}_{\mathbf{j}}^{\prime}, 1\right)$, and

$(k, \mathbf{l}, 1) \rightarrow(k, \mathbf{l}, 1)$.

It can be shown that the equations for the probabilities corresponding to the five transitions of the first set are, respectively,

$$
\begin{gathered}
p_{(k, 1,0),(k+1,1,1,0)}^{t}=\left(n-r_{k}-b_{\Sigma}+l_{\Sigma}\right) \\
\left(\frac{1-\varepsilon_{t}}{n}+\frac{\varepsilon_{t}}{n-r_{k}}\right)
\end{gathered}
$$

$$
\begin{gathered}
p_{(k, \mathbf{1}, 0),\left(k+1, \mathbf{l}+\mathbf{I}_{\mathbf{j}}^{\prime}, 0\right)}^{t}=\left(1-\rho_{j}\right)\left(b_{j}-l_{j}\right) \\
\left(\frac{1-\varepsilon_{t}}{n}+\frac{\varepsilon_{t}}{n-r_{k}}\right), \forall j
\end{gathered}
$$

$$
\begin{gathered}
p_{(k, \mathbf{l}, 0),\left(k+1, \mathbf{l}+\mathbf{I}_{\mathbf{j}}^{\prime}, 1\right)}^{t}=\rho_{j}\left(b_{j}-l_{j}\right) \\
\left(\frac{1-\varepsilon_{t}}{n}+\frac{\varepsilon_{t}}{n-r_{k}}\right), \forall j,
\end{gathered}
$$

$$
p_{(k, \mathbf{l}, 0),(k, \mathbf{l}, 0)}^{t}=\frac{1-\varepsilon_{t}}{n}\left(r_{k}-\sum_{j=1}^{h} \rho_{j} l_{j}\right)
$$

and

$$
p_{(k, \mathbf{l}, 0),(k, \mathbf{l}, 1)}^{t}=\left(\frac{1-\varepsilon_{t}}{n}\right) \sum_{j=1}^{h} \rho_{j} l_{j}
$$

for $(k, \mathbf{l}, 0) \in \Theta_{t}, t=0,1, \ldots, f-1$, such that $k \neq$ $n, 2 n, \ldots$, and those for the three transitions from the second set are 


$$
\begin{gathered}
p_{(k, \mathbf{b}, 0),(k+1, \mathbf{0}, 0)}^{t}=p_{(0, \mathbf{0}, 0),(1, \mathbf{0}, 0)}^{t}=\frac{n-b_{\Sigma}}{n}, \\
p_{(k, \mathbf{b}, 0),\left(k+1, \mathbf{I}_{\mathbf{j}}^{\prime}, 0\right)}^{t^{\prime}}=p_{(0, \mathbf{0}, 0),\left(1, \mathbf{I}_{\mathbf{j}}^{\prime}, 0\right)}^{t}= \\
\left(1-\rho_{j}\right) \frac{b_{j}}{n}, \forall j,
\end{gathered}
$$

and

$$
\begin{gathered}
p_{(k, \mathbf{b}, 0),\left(k+1, \mathbf{I}_{\mathbf{j}}^{\prime}, 1\right)}^{t}=p_{(0, \mathbf{0}, 0),\left(1, \mathbf{I}_{\mathbf{j}}^{\prime}, 1\right)}^{t}= \\
\rho_{j} \frac{b_{j}}{n}, \forall j,
\end{gathered}
$$

due to Equations 26, 27, and 28, respectively, for $t=n, n+1, \ldots, f-1$ and $k=n, 2 n, \ldots,\left[\frac{f-1}{n}\right]$, such that $k \leq t$. The last set of transitions to be considered, $(k, \mathbf{l}, 1) \rightarrow(k, \mathbf{l}, 1)$, merely signifies that the target has been perceived, thereby terminating the search. Clearly then,

$$
p_{(k, \mathbf{l}, 1),(k, \mathbf{l}, 1)}^{t}=1
$$

for $(k, \mathbf{l}, 1) \in \Theta_{t}, t=1,2, \ldots, f-1$.

Finally, it is not difficult to demonstrate from Equations 26 through 30 that

$$
\begin{gathered}
p_{(k, \mathbf{l}, 0),(k+1, \mathbf{l}, 0)}^{t}+\sum_{j=1}^{h} p_{(k, \mathbf{l}, 0),\left(k+1, \mathbf{l}+\mathbf{I}_{\mathbf{j}}^{\prime}, 0\right)}^{t}+ \\
\sum_{j=1}^{h} p_{(k, \mathbf{l}, 0),\left(k+1, \mathbf{l}+\mathbf{I}_{\mathbf{j}}^{\prime}, 1\right)}^{t}+p_{(k, \mathbf{l}, 0),(k, \mathbf{l}, 0)}^{t}+ \\
p_{(k, \mathbf{l}, 0),(k, \mathbf{l}, 1)}^{t}=1,
\end{gathered}
$$

for $(k, \mathbf{l}, 0) \in \Theta_{t}, t=0,1, \ldots, f-1$, such that $k \neq$ $n, 2 n, \ldots$, which is consistent with that fact that the system must transit from $(k, \mathbf{l}, 0)$ to either $(k+1, \mathbf{l}, 0)$, $\left(k+1, \mathbf{l}+\mathbf{I}_{\mathbf{j}}^{\prime}, 0\right),\left(k+1, \mathbf{l}+\mathbf{I}_{\mathbf{j}}^{\prime}, 1\right),(k, \mathbf{l}, 0)$, or $(k, \mathbf{l}, 1)$, and from Equations 31 through 33 that

$$
\begin{gathered}
p_{(k, \mathbf{b}, 0),(k+1, \mathbf{0}, 0)}^{t}+\sum_{j=1}^{h} p_{(k, \mathbf{b}, 0),\left(k+1, \mathbf{I}_{\mathbf{j}}^{\prime}, 0\right)}^{t}+ \\
\sum_{j=1}^{h} p_{(k, \mathbf{b}, 0),\left(k+1, \mathbf{I}_{\mathbf{j}}^{\prime}, 1\right)}^{t}=1
\end{gathered}
$$

for $t=n, n+1, \ldots, f-1$ and $k=n, 2 n, \ldots\left[\frac{f-1}{n}\right]$, such that $k \leq t$, which confirms that the system must transit from $(k, \mathbf{b}, 0)$ to either $(k+1, \mathbf{0}, 0),(k+$ $\left.1, \mathbf{I}_{\mathbf{j}}^{\prime}, 0\right)$ or $\left(k+1, \mathbf{I}_{\mathbf{j}}^{\prime}, 1\right)$. When Equation 34 is included, all of the transitions have been accounted for.

\section{ACKNOWLEDGMENTS}

This material is based upon work supported by the National Aeronautics and Space Administration (NASA) under Grant No. NCC 2-1288. Any opinions, findings, conclusions, or recommendations expressed in this material are those of the authors and do not necessarily reflect the views of NASA.

\section{REFERENCES}

Arani, T., Karwan, M. H., \& Drury, C. G. (1984). A variable-memory model of visual search. Human Factors, 26, 631-639.

Baveja, A., Drury, C. G., Karwan, M. H., \& Malon, D. M. (1996). Derivation and test of an optimum overlapping-lobes model of visual search. IEEE Transactions on Systems, Man, and Cybernetics - Part A: Systems and Humans, 26, 161-168.

Courtney, A. J., \& Guan, L. (1996). Visual performance with complete and incomplete visual lobe areas. Ergonomics, 39, 749-756.

Courtney, A. J., \& Guan, L. (1998). Assessing search performance with a simulation model. Human Factors and Ergonomics in Manufacturing, 8, 251-263.

Drury, C. G., \& Chi, C.-F. (1995). A test of economic models of stopping policy in visual search. IIE Transactions, 27, 382-393.

Eriksen, C. W. (1990). Attentional search of the visual field. In D. Brogan (Ed.), Visual search (pp. 3-19). London: Taylor \& Francis.

Gramopadhye, A. K., Drury, C. G., \& Prabhu, P. V. (1997). Training strategies for visual inspection. Human Factors and Ergonomics in Manufacturing, 7, 171-196.

Karwan, M. H., Morawski, T. B., \& Drury, C. G. (1995). Optimum speed of visual inspection using a systematic search strategy. IIE Transactions, 27, 291-299.

Krendel, E. S., \& Wodinsky, J. (1960). Visual search in unstructured fields. In A. Morris \& E. P. Horne (Eds.), Visual search techniques (pp. 151-169). Washington, DC: National Academy of Sciences.

Lin, T. (1991). A visual search model: The waiting time distribution of the number of fixations until detection. Naval Research Logistics, $38,157-170$

Megaw, E. D., \& Richardson, J. (1979). Target uncertainty and visual scanning strategies. Human Factors, 21, 303-315.

Morawski, T. B., Drury, C. G., \& Karwan, M. H. (1980). Predicting search performance for multiple targets. Human Factors, 22, $707-718$.

Morawski, T. B., Drury, C. G., \& Karwan, M. H. (1992). The optimum speed of visual inspection using a random search strategy. IIE Transactions, 24, 122-133.

Poulton, E. C. (1973). The effect of fatigue upon inspection work. Applied Ergonomics, 4, 73-83.

Ross, S. (2003). Introduction to probability models (8th ed.). San Diego, CA: Academic Press.

Sarac, A., Batta, R., \& Drury, C. G. (1997). One dimensional overlapping systematic search with finite length defect. In Sixth Industrial Engineering Research Conference Proceedings (pp. 107-112). Norcross, GA: Institute of Industrial Engineers.

Schoonard, J. W., \& Gould, J. D. (1973). Field of view and target uncertainty in visual search and inspection. Human Factors, 15, 33-42. 
Wang, M.-J. J., Lin, S.-C., \& Drury, C. G. (1997). Training for strategy in visual search. International Journal of Industrial Ergonomics, 20, 101-108.

Warner, H. D., \& Heimstra, N. W. (1972). Effects of noise intensity on visual target detection performance. Human Factors, 14, 181-185.

Wiener, E. L. (1975). Studies on visual search. In C. G. Drury \& J. G Fox (Eds.), Human reliability in quality control (pp. 31-43). London: Taylor \& Francis.

Williams, L. G. (1966). Target conspicuity and visual search. Human Factors, 8, 80-92.

Wolfe, J. M. (1994). Guided search 2.0: A revised model of visual search Psychonomic Bulletin and Review, 1, 202-238.

Brian J. Melloy is an associate professor of industrial engineering at Clemson University. He received his Ph.D. in operations research in 1986 from the University of South Florida.

Sourav Das is a Ph.D. candidate in industrial engineer- ing at Clemson University. He received his M.Tech. in quality, reliability, and operations research in 1998 from the Indian Statistical Institute, Calcutta.

Anand K. Gramopadhye is a professor and chair of industrial engineering at Clemson University. He received his Ph.D. in human factors in 1992 at the State University of New York-Buffalo.

Andrew T. Duchowski is an associate professor of computer science at Clemson University. He received his Ph.D. in computer science in 1997 at Texas A\&M University.

Date received: October 2, 2003

Date accepted: June 7, 2005 